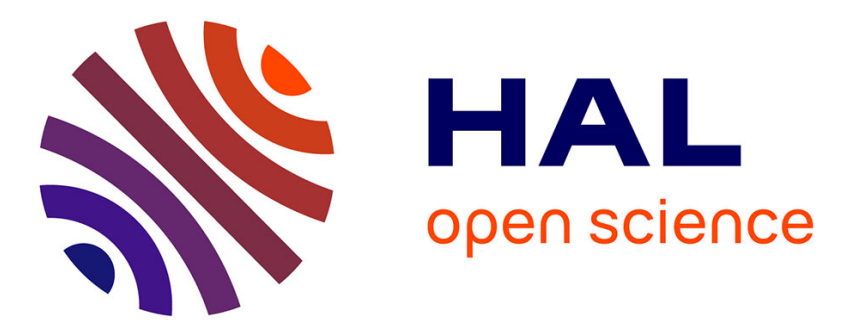

\title{
Impaired Performance of the Smash Stroke in Badminton Induced by Muscle Fatigue
}

Yann Le Mansec, Jérôme Perez, Quentin Rouault, Julie Doron, Marc Jubeau

\section{To cite this version:}

Yann Le Mansec, Jérôme Perez, Quentin Rouault, Julie Doron, Marc Jubeau. Impaired Performance of the Smash Stroke in Badminton Induced by Muscle Fatigue. International Journal of Sports Physiology and Performance, 2020, 15 (1), pp.52-59. 10.1123/ijspp.2018-0697 . hal-02521508

\section{HAL Id: hal-02521508 \\ https://hal-insep.archives-ouvertes.fr/hal-02521508}

Submitted on 27 Mar 2020

HAL is a multi-disciplinary open access archive for the deposit and dissemination of scientific research documents, whether they are published or not. The documents may come from teaching and research institutions in France or abroad, or from public or private research centers.
L'archive ouverte pluridisciplinaire HAL, est destinée au dépôt et à la diffusion de documents scientifiques de niveau recherche, publiés ou non, émanant des établissements d'enseignement et de recherche français ou étrangers, des laboratoires publics ou privés. 


\section{Impaired performance of the smash stroke in} badminton induced by muscle fatigue

$5 \quad$ Le Mansec Y1, Perez J1, Rouault Q1, Doron J1,2 \& Jubeau M1.

6 Affiliations

7 1 Laboratory Movement, Interactions, Performance (EA4334),

8 Faculty of Sport Sciences, University of Nantes, France

92 Laboratory of Sport, Expertise and Performance (EA7370),

10 French National Institute of Sport, Expertise and Performance,

11 Research Department, Paris, France

13 Corresponding author :

14 Marc Jubeau

15 Laboratoire "Motricité, Interactions, Performance" - EA 4334

16 Faculté des Sciences du Sport

17 Université de Nantes

1825 bis Boulevard Guy Mollet - BP 72206

1944322 Nantes cedex 3

20 France

21

22 Tel : $0033(0) 251837044$

23 Fax : $0033(0) 251837045$

24 E-mail : marc.jubeau@univ-nantes.fr

26 Running head : Fatigue and badminton smash stroke.

27 Abstract : 219 words

28 Text : 3462 words

29 Figures : 2 ; Tables : 2 
31 Muscle fatigue impairs the performance of the smash stroke in badminton

33 Abstract

34 Purpose: The main aim of the present study was to evaluate the 35 effects of muscle fatigue on badminton performance during a smash stroke. Methods: Seventeen young well-trained players completed twenty forehand smash twice (pre and post fatigue protocol) and both speed and precision of the strokes were 39 measured. The fatigue protocol consisted in ten series of ten 40 maximal counter movement jump (CMJ, 3 s rest in-between) 41 followed by eight lunges. Perception of effort and CMJ 42 performance during each series were also measured to assess 43 fatigue. Results: Shuttlecock speed decreased moderately ($443.3 \%)$ but significantly after the fatigue protocol $(\mathrm{P}<0.001$, $\left.45 \eta_{\mathrm{p} 2}=0.671\right)$. Precision significantly decreased after the fatigue 46 protocol $\left(-10.3 \%, \mathrm{P}=0.001, \eta_{\mathrm{p} 2}=0.473\right)$. The decrease in 47 precision was mainly due to an increased number of faults $48 \quad\left(\mathrm{P}=0.006, \eta_{\mathrm{p} 2}=0.378, \mathrm{~d}_{\mathrm{z}}=0.756\right)$ and to a decrease in accuracy $49 \quad\left(\mathrm{P}=0.066, \eta_{\mathrm{p} 2}=0.195, \mathrm{~d}_{\mathrm{z}}=0.478\right)$. Conclusion: The present study 50 showed that fatigue impairs the performance during specific 51 badminton skills. Moreover, by showing a slight decrease in 52 speed and a large decrease in accuracy of the shuttlecock when 53 fatigue is experienced, the present study suggested that, as 54 previously observed in other racket sports, the speed of the 55 missile appears to be the key factor used by the players to win 56 the rally. Coaches and physical trainers should therefore develop 57 intervention aiming to limit the negative impact of fatigue on 58 badminton strokes.

60 Keywords: sport motor skills, racket sports, speed, accuracy 
63 As a racket sport, it is well-known that performance in 64 badminton is multifactorial including physiological 65 psychological, technical and/or tactical parameters1. However, 66 the impact of each of these parameters on the ability to perform 67 at high-level is still a matter of debate. For instance, Ooi et al.2 68 did not observe differences between elite and sub-elite 69 Malaysian players when performing a battery of physical 70 performance tests, suggesting that other factors, such as 71 technical skills, were possibly of more importance2. This finding 72 is also supported by Chin et al.3, who found a low correlation 73 between a specific aerobic field-test and the ranking of the 74 players. By contrast, Phomsoupha and Laffaye 4 showed that the 75 speed of the shuttlecock evolved linearly with the level of the 76 players, allowing to differentiate high-skilled and elite players. 77 Similarly, Sakurai and Ohtsukis showed that the probability of 78 hitting a target, i.e. accuracy, was greater for the skilled players when compared to unskilled.

Neuromuscular fatigue, which can be defined as a loss of force (or power) production capacity accompanied by an increase in the effort required to perform the exercise 6 generally occurs during physical activity. As previously observed in other racket sports 7,8 , neuromuscular fatigue, demonstrated by a reduction of maximal voluntary contraction force, has been also reported after $1-\mathrm{h}$ of a simulated badminton game (-11\% and $-18 \%$ for the knee extensors and the knee flexors, respectively)9. Due to the high influence of technical parameters on performance in badminton, it is of critical importance to evaluate the impact of muscle fatigue on specific motor skills, which appears to be the most relevant parameters at high level2,3. In the setting of goaldirected movement assessed during racket sports, both speed and precision of the projectile are the two relevant technical parameters to describe the quality of a stroke $4,10,11$. To date, very few studies have attempted to emphasize the paradigm between the movement speed and the movement precision, i.e., speedaccuracy trade-off 12 , during the same task in a non-fatigued and 98 fatigued condition during racket sports 10,11,13.

99 Missenard et al.14 showed that muscle fatigue (elbow flexors and 100 extensors) impaired a pointing task by increasing the movement 101 duration to guarantee task success, i.e., precision. From an 102 ecological aspect, several studies have previously investigated 103 the impact of muscle fatigue on specific sport motor skills by 104 measuring simultaneously these parameters during racket sports. 105 These studies demonstrated that precision is largely affected in 106 fatigued condition in tennis 11 and table tennis 15 whereas ball 107 speed is less deteriorated. However, this result was not 108 systematic13. To the best of our knowledge, no study has 
109 attempted to evaluate the impact of muscle fatigue on sport

110 motor skills during a specific badminton task.

111 This study aimed therefore to evaluate the impact of muscle

112 fatigue on badminton-specific technical performance. To do that,

113 we have first developed a field test to evaluate the reliability for

114 both precision and shuttlecock speed during a smash stroke,

115 which is classically considered as the most powerful stroke,

116 generally used for winning the rally16 (Study 1). Thereafter, this

117 test was performed before and after a fatiguing protocol (Study

118 2) to measure in what extent fatigue could impair the speed

119 and/or the accuracy of the shuttlecock during a specific

120 badminton stroke.

122 METHODS

\section{Participants}

124 Fifty-five participants (mean \pm SD age: $22.7 \pm 5.7 \mathrm{yr}$; 125 height: $179.9 \pm 6.9 \mathrm{~cm}$; mass: $71.9 \pm 6.2 \mathrm{~kg}$ ) volunteered to

126 participate in this study. All participants or legal representatives

127 for minors gave their written consent after being informed about

128 the procedures of the protocols, which were approved by local

129 ethics committee of Nantes, in accordance with the Declaration

130 of Helsinki. Among these players, thirty-eight (males only, mean $131 \pm$ SD age: $25.3 \pm 5.0 \mathrm{yr}$; height: $181.2 \pm 5.5 \mathrm{~cm}$; mass: $74.1 \pm 4.8$

$132 \mathrm{~kg}$ ) participated in Study 1, i.e., reproducibility of the specific

133 test, and seventeen $(\mathrm{M}=11, \mathrm{~F}=6,17.0 \pm 0.8 \mathrm{yr}, 177.0 \pm 8.8 \mathrm{~cm}$,

$13467.1 \pm 6.4 \mathrm{~kg}$ ) participated in Study 2, i.e., effects of muscle

135 fatigue on badminton-specific technical performance.

136 Study design

\section{Study 1}

138 Players were divided into four groups according to their single

139 ranking in the French Federation of Badminton (FFBad) and

140 their training volume (Table 1). In the high trained group (HT,

$141 \mathrm{n}=9)$, all players were high-trained $(4.9 \pm 1.3$ times per week)

142 and participated in national competitions. In the moderate

143 trained group (MT, n=10), players were moderate to well-trained

144 (3.1 \pm 1.0 times per week) and participated in a regional

145 championship. In the low trained group (LT, n=9), players were

146 less trained (2.0 \pm 0.7 times per week) and participated in a local

147 championship. The untrained group (UT, n=10) was composed

148 by sports-science-students, without experience in badminton and

149 not ranked by the FFBad.

150 After a 15-min standardized warm-up and once familiarized (10

151 trials) with the procedures, all the participants performed one 
session which consisted of performing the specific test (see specific test) twice (intra-session reliability), with a 10-min resting period in between. Thirty-three participants, among the 38 participants initially recruited, participated in a second session to assess the inter-session reliability. A minimum of 7 days interval was defined between both sessions.

Study 2

All participants $(n=17)$ were recruited from French National badminton training centers and were well-trained $(15.5 \pm 1.8$ h.wk-1), regularly participated in national competitions in the French championship, and none of them had any known muscle/tendon injury for at least one year. One session (duration: $\sim 45 \mathrm{~min}$ ) was performed to assess the effects of muscle fatigue on badminton-specific technical performance. All sessions took place in a homologated specific facility comprising two courts. All participants were familiarized with all procedures at the beginning of the session. Before and immediately after $(\sim 30 \mathrm{~s})$ completion of the fatigue protocol (see below for further details) participants performed the specific badminton test (see specific test) to measure the quality (precision and speed of the shuttlecock) of the smash. To control for the effect of the fatiguing protocol, both subjective (perception of effort) and objective (maximal height during a countermovement jump $[\mathrm{CMJ}])$ measurements of fatigue were assessed.

176 We quantified badminton-specific technical performance by measuring stroke parameters during the specific test previously described (Study 1). The test took place in a second court, close to the one used during the fatigue protocol. To take into account the ecological aspects of badminton smash 17 , male players were asked to jump when performed the stroke while this rule was not mandatory for the female players.

\section{Specific test}

The test consisted in 20 forehand overhead smash, which is considered as a typical offensive stroke. They were asked to hit, alternatively, two targets placed on the court (Fig 1A). The 2 rectangular targets $(212 \mathrm{~cm}$ length $/ 40 \mathrm{~cm}$ width) were positioned on the sides and were divided into 2 areas $(212 \mathrm{~cm}$ length $/ 20 \mathrm{~cm}$ width; Fig 1A). The players were instructed to hit the shuttlecock (Aerosensa 50, Yonex®, Tokyo, Japan) for winning the rally as they do during an official game. Ten trials were evaluated for

193 Badenko®, Pampelonne, France) to ensure protocol 194 standardization and repeatability for shuttlecock speed, 195 trajectory and placement. For each trial, the robot sent the 196 shuttlecock to the center of the court, placing it 75-80 cm away 197 from the baseline (Fig 1A). The shuttlecock was delivered by the 
robot every $3 \mathrm{~s}$ with a rising trajectory. New shuttlecocks were used for each session and were replaced when they were damaged.

201 The performance assessment was explained to the participants 202 before starting the test. During the test, participants were 203 strongly encouraged and informed on their outcome in order to 204 maintain vigilance and concentration throughout the procedure.

\section{Muscle fatigue}

206 To induce muscle fatigue (study 2), participants completed 10 series of two specific movements frequently used by the badminton players, i.e., $\mathrm{CMJ}_{17}$ and lunges9,17,18. Each series consisted of ten CMJ performed every $3 \mathrm{~s}$. CMJ were realized hands akimbo, and subjects were asked to perform each trial maximally. Once the CMJ performed, subjects kept their own racket and had to realize 8 lunges with no rest between the two exercises. During the lunges task, participants were asked to touch alternatively the right and the left parts of the net with their racket (1-m width). Between two lunges, participants moved 1$\mathrm{m}$ behind the short service line before continuing the exercise (Fig 1B). Once the exercise completed, subjects came back walking (10-s) to the baseline, rated their perceived exertion, and started a new series, until 10 series were performed. During the last series, subjects only performed $10 \mathrm{CMJ}$, without execution of lunges to better assess the fatigue induced by our protocol, since CMJ represents the objective measure of fatigue used in this study. The instruction was given to the players to directly move on the badminton test after the last series of CMJ. This protocol was chosen to induce a level of fatigue consistent with the one observed during a real badminton game. We assume that it represented a good compromise, taking into account the duration of the protocol $(\sim 7 \mathrm{~min})$, the number of lunges and jumps performed9. To maintain motivation, subjects were strongly encouraged during the entire protocol.

\section{Data processing}

\section{Shuttlecock speed}

234 For each smash, the speed of the shuttlecock hit by the player was measured with a radar (Stalker ATS ii, Stalker Radar®,

236 Plano, Texas, USA) at a frequency of $50 \mathrm{~Hz}$ and an accuracy of $237 \pm 0.041 \mathrm{~m} . \mathrm{s}-1$. The radar was located $3 \mathrm{~m}$ behind the player, at a 238 height of $2.50 \mathrm{~m} 4$. To ensure recording of the speed data, an 239 experimenter manually pointed the radar towards the area 240 targeted by the player. All data were recorded on a personal 241 laptop (software: Stalker ATS 5.0, Plano, Texas, USA). The 242 shuttlecock speed for each of the 20 smashes was measured. The 
mean speed was then calculated by averaging the 20 speed values.

\section{Precision}

246 The evaluation of precision was assessed by using a video camera (AHD-H12 VAZ2S, Aiptek®, Rowland Street, Ca, USA) for post-processing analysis. The camera was located $5 \mathrm{~m}$ behind the baseline opposite to the player and in the axis of the radar and the robot (Fig 1A). The following procedure was used: when the shuttlecock reached the part of the target nearest from the sideline, 3 points were granted (i.e., accuracy); 2 points when the shuttlecock reached the part of the target furthest from the sideline (i.e., consistency); 1 point when the shuttlecock reached the court but did not touch the targets (i.e., neutral); 0 point when a fault was committed (Fig 1A). This procedure gave a score between 0 and 60 for each series.

Perception of effort (RPE). In study 2, perception of effort, defined as "the conscious sensation of how hard, heavy, and strenuous the physical task is" 20 was measured throughout the fatigue protocol. Participants were asked to rate the effort related to the physical task just performed by using the 6-20 Borg Scale21 after the first nine series.

264 CMJ. In study 2, optojump photoelectric cells (Microgate, 265 Bolzano, Italy) were used to precisely measure the jump 266 height22. Fatigue was assessed by calculating the average of the height of the $10 \mathrm{CMJ}$ performed from each series. We also compared CMJ performance between the average of the first three CMJs of the first series (trials 1-3) and the last three CMJs of the last series (trials 98-100). All data were recorded on a personal laptop with the appropriate software (Optojump software, version 3.01.0001) for further treatment.

\section{Statistical analysis}

275 Statistical tests were performed with Statistica ${ } V 6$ software 276 (Statsoft, Tulsa, USA) and G*Power® software (version 3.1.6 277 Universität Düsseldorf, Germany). Assumptions of normality 278 (Kolmogorov-Smirnov test) and sphericity (Mauchly test) of 279 data were checked as appropriate. Values are presented as mean $280 \pm$ SD.

\section{Study 1}

282 Speed and precision were compared across the four populations 283 by using a one-way analysis of variance (ANOVA) (4 between284 subjects factors) for the first series of the first day. Correlation 285 analyses (Bravais-Pearson) were performed to determine 
whether speed, precision, or performance index were correlated to the skill level (i.e., FFBad ranking). For each group, standard error of measurements (SEM), intraclass correlation coefficients (ICC) and coefficient of variation (CV) were calculated for both intra-day and inter-day23. Since we used a set time between sessions, the ICC $(3,1)$ was chosen from Shrout and Fleiss 24. Inter-day reliability was assessed by using the first series of day

$2931 v s$. the first series of day 2.

\section{Study 2}

295 RPE and CMJ height were tested using one-factor (time) 296 ANOVA with repeated measures. Stroke smash parameters 297 (speed and precision) and the distribution, i.e., percentage of 298 faults, neutral, consistency and accuracy, were then tested with 299 one-way (time) ANOVA.

301 In both studies, the level of significance was set at $\mathrm{P}<0.05$ and 302 post-hoc analyses were performed when appropriated using 303 HSD Tukey test for multiple comparisons. For the main effects 304 of the ANOVAs, partial eta square $\left(\eta_{\mathrm{p} 2}\right)$ are reported, with 305 moderate and large effects considered for $\eta_{\mathrm{p} 2} \geq 0.07$ and $\geq 0.14$, 306 respectively25. For the follow up tests, Cohen's effect sizes $d$ are 307 reported, with small, moderate and large effects considered for $d$ $308 \geq 0.2, \geq 0.5$ and $\geq 0.8$, respectively 25 .

\section{RESULTS}

\section{Study 1}

\section{Effect of expertise on performance factors}

\section{Shuttlecock speed}

314 Our results showed a significant effect of expertise $\left(\eta_{\mathrm{p} 2}=0.954\right.$;

$315 \mathrm{P}<0.001)$. Shuttlecock speed was higher for HT $(158.1 \pm 5.5$

316 km.h-1) compared to MT, LT and UT (136.4 \pm 4.0 km.h-1, 121.0

$317 \pm 4.2 \mathrm{~km} . \mathrm{h}-1$ and $101.7 \pm 5.3 \mathrm{~km} . \mathrm{h}-1, d$ ranged from 4.51 to 10.44 ,

318 respectively; $\mathrm{P}<0.001)$, for $\mathrm{MT}$ compared to $\mathrm{LT}$ and UT ( $d$

319 ranged from 3.79 to 7.42 , respectively, $\mathrm{P}<0.001$ ), and LT

320 compared to UT $(d=4.04, \mathrm{P}<0.001)$. In addition, a significant

321 correlation was observed between the speed of the shuttlecock

322 and the ranking of the player $(\mathrm{r}=-0.81 ; \mathrm{P}<0.001 ; \mathrm{n}=28$, UT

323 excluded). 
A significant main effect was observed for precision $\left(\eta_{\mathrm{p} 2}=0.849\right.$; $\mathrm{P}<0.001)$, showing that the score of precision (/60) was higher for HT $(33.1 \pm 2.5)$ compared to MT, LT and UT $(26.1 \pm 2.4$, $21.3 \pm 1.7$ and $18.4 \pm 3.0, d$ ranged from 2.86 to 5.52 , respectively; $\mathrm{P}<0.001$ ), for $\mathrm{MT}$ compared to $\mathrm{LT}$ and UT (d ranged from 2.26 to 2.85 , respectively; $\mathrm{P}=0.001$ and $\mathrm{P}<0.001$, respectively). A significant correlation was also found between precision and ranking of the players $(\mathrm{r}=-0.78 ; \mathrm{P}<0.001 ; \mathrm{n}=28$, UT excluded).

\section{Intra- and inter-session reliability}

The mean values, SD, ICC, SEM and CV are shown in Table 2 for intra- and inter-session reliability for each group. Low CV were found for HT among all parameters, with low to high ICC. The CV for MT group were also acceptable for all variables and low to high ICC were also observed. The CV for LT were acceptable, and low to high ICC were found. Except for the speed of the shuttlecock, CV were generally higher for UT for intra- and inter-session, as well as ICC varied widely depending on the parameter considered.

Study 2

\section{Effects of the fatigue protocol}

\section{Perception of effort.}

There was a significant effect of time on the perceived exertion $\left(\mathrm{P}<0.001, \eta_{\mathrm{p} 2}=0.852\right)$. RPE progressively increased throughout the fatigue protocol (from 12.0 \pm 1.7 after the first series to 18.8 \pm 0.8 after the 9 th series $)$.

There was a main effect of time on the height performed during the CMJ $\left(-10.1 \pm 6.8 \%, \mathrm{P}<0.001, \eta_{\mathrm{p} 2}=0.468\right.$, Fig 2$)$. The best performance was measured after the first series $(32.4 \pm 4.6 \mathrm{~cm})$ while the weakest performance was measured after the last series $(29.0 \pm 4.0 \mathrm{~cm})$. The greatest difference was observed between the first three CMJs of the first series $(32.9 \pm 4.6 \mathrm{~cm})$ and the last three CMJs of the last series $(27.4 \pm 4.5 \mathrm{~cm})$, i.e. $-16.9 \pm 9.8 \%$ $\left(\mathrm{d}_{\mathrm{z}}=1.692\right.$, Fig 2).

\section{Effects of muscle fatigue on badminton performance}

\section{Shuttlecock speed.}

There was a significant effect of time for the shuttlecock speed $\left(\mathrm{P}<0.001, \eta_{\mathrm{p} 2}=0.671\right)$. This parameter decreased about $3.3 \pm$ $2.4 \%$ in the fatigued state, from $151.2 \pm 4.6 \mathrm{~km} . \mathrm{h}-1$ to $146.2 \pm$ 3.9 km.h-1 (Fig 3A). 
367 There was a significant effect of time for the total score $368\left(\mathrm{P}=0.001, \eta_{\mathrm{p} 2}=0.473\right)$. This parameter decreased about $10.3 \pm$ $36910.3 \%$ in the fatigued state, from $27.4 \pm 5.9$ to $24.4 \pm 4.7$ (Fig $3703 \mathrm{~B})$.

\section{Distribution.}

372 There was a main effect of time on the number of faults 373 committed by the players (from $29.7 \pm 13.3 \%$ to $37.9 \pm 11.6 \%$, $\left.374 \mathrm{P}=0.006, \eta_{\mathrm{p} 2}=0.378, \mathrm{~d}_{\mathrm{z}}=0.756\right)$. There was no main effect of time 375 on consistency (from $19.7 \pm 13.0 \%$ to $20.0 \pm 10.2, \mathrm{P}=0.918$, $\left.376 \eta_{\mathrm{p} 2}=0.0006, \mathrm{~d}_{\mathrm{z}}=0.026\right)$ while a clear trend was observed for 377 accuracy, with small to moderate effect size (from $23.5 \pm 8.8 \%$ 378 to $19.7 \pm 6.0 \%, \mathrm{P}=0.066, \eta_{\mathrm{p} 2}=0.195, \mathrm{~d}_{\mathrm{z}}=0.478$, Fig $3 \mathrm{C}$ ).

\section{DISCUSSION}

380 The main outcomes of the present study showed that the quality of the smash was impaired by muscle fatigue. The speed of the shuttlecock slightly decreased while the precision substantially decreased after the fatigue protocol.

Specific test

385 The results of our study 1 demonstrated that the test proposed 386 herein (i) is sensitive enough to discriminate groups or players with different playing levels, (ii) shows that, when the shuttlecock parameters (speed and precision) are assessed simultaneously, they are both correlated with the ranking of the player and (iii) is highly reliable (intra and intersession) when high trained players are considered, as used in study 2 . Specifically, as regards shuttlecock speed, we observed high ICC for all groups (around 0.8 for intra-session reliability), whereas ICC varied from low to very-low (ranged from 0.15 to 0.42 ) for the precision. However, a low between-subject variability is known to decrease ICC23,26,27. In the current study, we observed a very small intra-session variability for both high and moderate trained groups (i.e. SD ranged from 1.2 to 2.5). As both CV and SEM were low, we considered that the test proposed herein was consistent for the players who exhibited sufficient technical skills. Thus, the use of this specific test was relevant to assess the effects of fatigue on the quality of the smash parameters.

\section{Markers of fatigue}

Psychological marker. The perceived exertion regularly increased throughout the fatigue protocol, showing that the players were close to exhaustion before performing the second badminton test. Although it should be kept in mind that RPE measured during official junior games is less than that induced 
in the current study ( 14.5 vs 18.8$) 19$, it is plausible that after a

410 particularly challenging rally, the perceived exertion is

411 significantly greater than that measured at the end of the game.

412 Thus, Abian et al.28 showed that in the modern badminton, the

413 rally duration tends to increase, as well as the recovery between

414 two rallies, reflecting a more demanding physical condition.

415 Therefore, although the perceived exertion measured at the end

416 of the fatigue protocol was very high, we assume that such a

417 fatigue may occasionally exist during badminton game.

418 Physical marker. The increase in perceived exertion was 419 accompanied by a significant decrease in performance to 420 perform CMJ $(-10.1 \pm 6.8 \%)$. This decrease was mainly due to 421 the repetition of lunges and CMJ without rest during $\sim 7 \mathrm{~min}$, 422 which could have induced muscle damage. Abian-Vicen et al.29 423 have previously shown that performance in CMJ decreased over 424 time during successive badminton games $(-7.2 \%, \mathrm{P}<0.05)$, 425 although no effect was reported after only one game.

426 Taken together, both subjective and functional parameters, allow 427 to conclude that the fatigue protocol successfully induced a state 428 of fatigue in our subjects.

\section{$429 \quad$ Fatigue impairs specific badminton skill}

430 In the current study, we observed that fatigue phenomenon, assessed by both subjective (increased RPE) and functional (decreased CMJ performance) parameters, induced a moderate but significant decrease of the speed of the shuttlecock during a smash stroke $(-3.3 \pm 2.4 \%)$. Such a result has previously been reported in tennis 13 while Le Mansec et al.15 did not found any decrease of this parameter after both upper and lower limb fatigue during table tennis stroke. Yet, by reporting significant differences in shuttlecock speed during the smash stroke depending on the level of the players, previous 4 and current (Study 1) studies demonstrated that this parameter was very discriminant to perform at a high level during a badminton game. Thus, despite their fatigue level, the players tried to maintain, consciously or unconsciously, a high shuttlecock speed during the second specific test.

445 We also found that the precision was significantly decreased 446 during the second badminton specific test, i.e., after the fatigue protocol. This decrease in precision was mainly due to an increase in the number of faults and, to a lesser extent, to a decrease in accuracy. Thus, although Missenard et al.14 showed that during a pointing task, fatigued participants decreased the speed of their movement in order to maintain their task success, the present study gave evidence that, during a specific badminton motor skill, players have given priority to the speed of the shuttlecock, even if this led to commit more faults or reach the 
target less often. This result has already been observed in racket sports such as tennis11 and table tennis 15 .

457 How fatigue impairs stroke performance during 458 badminton smash?

459 Since the design of the current study was mainly descriptive, we

460 did not have access to the underlying mechanisms, which were 461 able to explain the results observed. However, based on previous 462 studies, we can advance some hypotheses. Indeed, Sakurai and 463 Ohtsukis showed that high skilled badminton players had 464 consistent motor program, i.e., reliability of the muscle activity 465 pattern, when they performed badminton smash. This ability to 466 perform consistently results in a lower unforced errors5, which 467 has a great influence in winning or losing the game30. However, 468 the coordination may be affected and a shift in motor control can 469 be observed when fatigue is experienced, leading to conflicting 470 results 11,31,32. For instance, despite changes in technical factors 471 induced by muscle fatigue, speed and accuracy were unchanged 472 during a specific water polo motor skill32. As regards table 473 tennis, Aune et al.31 suggested that, when fatigued, high skilled 474 players adjusted the motor coordination strategy to maintain 475 accuracy, as explained by Missenard et al.14. Conversely, Rota 476 et al.11 showed that fatigue in upper limb muscles induced a 477 decrease in forehand stroke accuracy associated with changes in 478 muscle activation level, while the speed of the ball was 479 maintained. Even if muscle activation was not measured in the 480 current study, it might be speculated that changes in the level of 481 activation of fatigued muscles could partly explain our results. 482 However, further studies are required to better understand the 483 biomechanical origins (e.g. muscular coordination pattern) of the 484 decrease in smash stroke performance observed herein.

485 It is classically accepted that the temporal structure, the 486 notational analysis or the physiological characteristics may vary 487 depending on the gender, suggesting a higher demand during the 488 games for males17. This aspect is reinforced by the study of 489 Fernandez-Fernandez et al.19, who showed that the activity 490 pattern of the match is different between young males and young 491 females players (16 yr), but induced only slight different 492 physiological responses, i.e. same heart rate, blood lactate 493 response, RPE. Consequently, it could be possible that the 494 protocol used in the present study to induce fatigue may affect 495 female players in a different manner than male players and 496 change our results. However, no gender differences appeared for 497 the markers of fatigue (RPE: $18.9 \pm 0.7$ vs $18.6 \pm 1.0$; CMJ: $498 \quad 10.4 \pm 4.6 \%$ vs $-9.4 \pm 10.3 \%$ for males and females, 499 respectively). Similarly, no gender difference was observed for 500 the decrement in speed of the shuttlecock $(-3.4 \pm 2.0 \%$ vs $-3.0 \pm$ $5013.1 \%$ ). Only the decrease of the precision was different, i.e. 502 greater for males $(-13.2 \pm 8.0 \%)$, when compared to females (- 
$5035.1 \pm 12.7 \%$ ), possibly due to the fact that females do not jump

504 for smash. Taken as a whole, gender seems to have a little

505 influence on our results.

\section{Practical applications}

507 The results of the current study, i.e., decrease in both speed 508 (moderate) and precision (large effect) of the shuttlecock

509 following a fatiguing protocol, are relevant for coaches and

510 physical trainers, since the number of unforced errors is an 511 important factor to win the game 30 and the speed of the missile 512 is also determinant to perform at high level4. Thus, coaches, 513 physical and/or mental trainers should accustom badminton 514 players to experience fatigue during training and to propose 515 relevant training programs in order to minimize the magnitude 516 of the deleterious effect of fatigue during the game.

\section{CONCLUSION}

519 This study highlighted that fatigue impairs the quality of the

520 stroke performance in badminton, by altering the speed $(\sim 3 \%)$

521 and the precision $(\sim 10 \%)$ of the shuttlecock. Moreover, our 522 results suggest that badminton coaches should improve the 523 physical state of their athletes to postpone the negative effects of 524 fatigue on specific technical skills.

526 Acknowledgments

527 The study was supported by grants from the French Ministry of 528 Sports (contract no. 15r16). The authors thank Claude Leveau 529 for his precious advices and Olivier Bime and the French 530 Badminton Federation (FFBad) as well as Mourad Amrani, 531 Frederic Pottier, the League of Badminton of Pays de la Loire 532 and Badenko® for her material and human assistance. The 533 authors also thank all the badminton players. 
537 1. Lees A. Science and the major racket sports: a review. $J$.

538 Sports Sci. 2008;21:707-732.

539 2. Ooi CH, Tan A, Ahmad A, Kwong KW, Sompong R,

540 Ghazali KA, Liew SL, Chai WJ, Thompson MW. Physiological

541 characteristics of elite and sub-elite badminton players. J Sports

542 Sci. 2009;27:1591-1599.

543 3. Chin MK, Wong AS, So RC, Siu OT, Steininger K, Lo

544 DT. Sport specific fitness testing of elite badminton players. $\mathrm{Br}$

$545 \quad J$ Sports Med. 1995;29:153-157.

546 4. Phomsoupha M, Laffaye G. Shuttlecock velocity during

547 a smash stroke in badminton evolves linearly with skill level.

548 Computer Methods in Biomechanics and Biomedical 549 engineering. 2014;17(Suppl. 1):140-141.

5505 5. Sakurai S \& Ohtsuki T. Muscle activity and accuracy of

551 performance of the smash stroke in badminton with reference to

552 skill and practice. J Sports Sci. 2000;18:901-914.

553 6. Williams CA \& Ratel S. Definitions of muscle fatigue.

554 In: Williams CA, Ratel S, editors. Human Muscle Fatigue. New

555 York (NY): Routledge; 2009:3-16.

556 7. Girard O, Lattier G, Micallef J-P, Millet GP. Changes in

557 exercise characteristics, maximal voluntary contraction, and

558 explosive strength during prolonged tennis playing. Br J Sports

559 Med. 2006;40:521-526.

560 8. Le Mansec Y, Sève C, Jubeau M. Neuromuscular fatigue

561 and time motion analysis during a table tennis competition. $J$

562 Sports Med Phys Fitness. 2017;57:353-361.

5639 . Lin Z. Neuromuscular fatigue following a singles

564 badminton match. Master thesis, Edith Cowan University. 2014.

565 10. Le Mansec Y, Dorel S, Nordez A, Jubeau M. Sensitivity

566 and reliability of a specific test of stroke performance in table

567 tennis. Int J Sports Physiol Perfor. 2016;11:678-684.

568 11. Rota S, Morel B, Saboul D, Rogowski I, Hautier C.

569 Influence of fatigue on upper limb muscle activity and

570 performance in tennis. J Electromyogr Kinesiol. 2014;24:90-97.

571 12. Fitts PM. The information capacity of the human motor

572 system in controlling the amplitude of movement. J Exp Psychol.

573 1954;47:381-391.

574 13. Vergauwen L, Spaepen AJ, Lefevre, Hespel P. 575 Evaluation of stroke performance in tennis. Med Sci Sports 576 Exerc. 1998;30:1281-1288.

577 14. Missenard O, Mottet D, Perrey S. Adaptation of motor 578 behavior to preserve task success in the presence of muscle 579 fatigue. Neuroscience. 2009;161:773-786. 
580 15. Le Mansec Y, Pageaux B, Nordez A, Dorel S, Jubeau M.

581 Mental fatigue alters the speed and the accuracy of the ball in

582 table tennis. J Sports Sci 2018;36:2751-2759.

583 16. Abian-Vicen J, Castanedo A, Abian P, Sampedro J.

584 Temporal and notational comparison of badminton matches

585 between men's singles and women's singles. Int. J. Perform.

586 Analysis in Sport 2013;13:310-320.

587 17. Phomsoupha M, Laffaye G. The science of badminton:

588 game characteristics, anthropometry, physiology, visual fitness

589 and biomechanics. Sports Med. 2015;45:473-495.

590 18. Kuntze G, Mansfield N, Sellers W. A biomechanical 591 analysis of common lunge tasks in badminton. J Sports Sci. 592 2010;28:183-191.

593 19. Fernandez-Fernandez J, de la Aleja Tellez JG, Moya-

594 Ramon M, Cabello-Manrique D, Mendez-Villanueva A. Gender 595 differences in game responses during badminton match play. $J$ 596 Strength Cond Res. 2013;27:2396-2404.

597 20. Marcora S. Effort. Perception of. In E. B. Goldstein, ed. 598 Encyclopedia of perception. Thousand Oaks, CA: SAGE 599 Publications Inc; 2010:380-383.

600 21. Borg G. Borg's perceived exertion and pain scales. 601 Champaign, IL: Human Kinetics; 1998.

602 22. Glatthorn JF, Gouge S, Nussbaumer S, Stauffacher S, 603 Impellizzeri FM, Maffiuletti NA. Validity and reliability of 604 Optojump photoelectric cells for estimating vertical jump height.

605 J Strength Cond Res. 2011;25:556-560.

606 23. Hopkins WG. Measures of reliability in sports medicine 607 and science. Sports Med 2000;30:1-15.

608 24. Shrout PE, Fleiss JL. Intraclass correlations: uses in 609 assessing rater reliability. Psychol Bull. 1979;86:420-428.

610 25. Cohen J. Statistical Power Analysis for the Behavioral

611 Sciences. New York: Academic press; 2013.

612 26. Atkinson DG \& Nevill AM. Statistical methods for 613 assessing measurement error (reliability) in variables to. Sports 614 Med. 2012;26:217-238.

615 27. Weir JP. Quantifying test-retest reliability using the 616 intraclass correlation coefficient and the SEM. J. Strength Cond. 617 Res. Natl. Strength Cond. Assoc. 2005;19:231-240.

618 28. Abián P, Castanedo A, Feng XQ, Sampedro J, Abian619 Vicen J. Notational comparison of men's singles badminton 
620 matches between Olympic games in Beijing and London. Int. J.

621 Perform. Analysis in Sport. 2014;14:42-53.

622 29. Abian-Vicen J, Castanedo A, Abian P, Gonzalez-Millan

623 C, Salinero J, del Coso J. Influence of successive badminton 624 matches on muscle strength, power, and body-fluid balance in 625 elite players. Int J Sports Physiol Perfor. 2013;9:689-694.

626 30. Cabello Manrique D, González-Badillo JJ. Analysis of 627 the characteristics of competitive badminton. Br J Sports Med. $628 \quad 2003 ; 37: 62-66$.

629 31. Aune TK, Ingvaldsen RP, Ettema GJ. Effect of physical 630 fatigue on motor control at different skill levels. Percept Mot 631 Skills. 2008;106:371-386.

632 32. Royal KA, Farrow D, Mujika I, Halson SL, Pyne D, 633 Abernethy B. The effects of fatigue on decision making and 634 shooting skill performance in water polo players. J Sports Sci. 635 2006;24:807-815.

636

637 
639 Table 1. Anthropometric values and badminton experience for untrained (UT), low trained (LT), moderate trained (MT) and high trained group 640 (HT) for the participants of study 1 . Values are mean \pm SD.

\begin{tabular}{ccccc} 
& UT & LT & MT & HT \\
\cline { 2 - 5 } Age (years) & 10 & 9 & 10 & 9 \\
Height (cm) & $19.2 \pm 0.8$ & $30.3 \pm 3.9$ & $27.4 \pm 2.8$ & $24.8 \pm 2.9$ \\
Body mass (kg) & $70.4 \pm 3.0$ & $77.7 \pm 5.5$ & $73.2 \pm 3.7$ & $75.4 \pm 4.2$ \\
Badminton experience & $/$ & $13.5 \pm 3.5$ & $18.3 \pm 2.9$ & $17.0 \pm 3.1$ \\
(years) & & & & \\
Training volume (h.w-1) & $/$ & $3.2 \pm 1.3$ & $5.5 \pm 2.3$ & $8.4 \pm 1.3$ \\
Ranking (FFBad) & $/$ & $7440.3 \pm 2144.7$ & $1782.4 \pm 539.0$ & $196.0 \pm 133.8$ \\
\hline
\end{tabular}




\begin{tabular}{|c|c|c|c|c|c|c|c|c|c|c|c|c|}
\hline \multirow{3}{*}{$\begin{array}{c}\text { Dependent } \\
\text { variables }\end{array}$} & \multicolumn{6}{|c|}{ Intra-session reliability } & \multicolumn{6}{|c|}{ Inter-session reliability } \\
\hline & \multirow[t]{2}{*}{$\mathbf{n}$} & \multicolumn{2}{|c|}{$\begin{array}{l}\text { Mean value } \\
\text { (SD) }\end{array}$} & \multirow[t]{2}{*}{ ICC } & \multirow[t]{2}{*}{$\begin{array}{c}\text { Mean CV } \\
\text { (SD) }\end{array}$} & \multirow[t]{2}{*}{ SEM } & \multirow[t]{2}{*}{$\mathbf{n}$} & \multicolumn{2}{|c|}{$\begin{array}{l}\text { Mean value } \\
\text { (SD) }\end{array}$} & \multirow[t]{2}{*}{ ICC } & \multirow[t]{2}{*}{$\begin{array}{c}\text { Mean CV } \\
\text { (SD) }\end{array}$} & \multirow[t]{2}{*}{ SEM } \\
\hline & & T1 & $\mathbf{T 2}$ & & & & & T1 & T3 & & & \\
\hline HT & 9 & & & & & & 9 & & & & & \\
\hline Shuttlecock speed (km.h-1) & & $158.1 \pm 5.5$ & $159.0 \pm 6.3$ & 0.81 & $1.7 \pm 0.5$ & 3.0 & & $158.1 \pm 5.5$ & $159.9 \pm 7.1$ & 0.63 & $2.4 \pm 0.8$ & 4.2 \\
\hline Precision (/60) & & $33.1 \pm 2.5$ & $32.2 \pm 1.3$ & 0.18 & $4.8 \pm 3.0$ & 1.8 & & $33.1 \pm 2.5$ & $32.7 \pm 2.6$ & 0.62 & $3.8 \pm 3.4$ & 1.7 \\
\hline MT & 10 & & & & & & 8 & & & & & \\
\hline Shuttlecock speed (km.h-1) & & $136.4 \pm 4.0$ & $138.5 \pm 5.0$ & 0.86 & $1.6 \pm 0.6$ & 1.9 & & $136.4 \pm 3.4$ & $138.6 \pm 4.4$ & 0.48 & $2.3 \pm 0.6$ & 3.0 \\
\hline Precision (/60) & & $26.1 \pm 2.4$ & $25.6 \pm 1.2$ & 0.15 & $6.3 \pm 2.2$ & 1.8 & & $25.5 \pm 2.3$ & $24.8 \pm 3.2$ & 0.48 & $7.9 \pm 3.6$ & 2.2 \\
\hline $\mathbf{L T}$ & 9 & & & & & & 6 & & & & & \\
\hline Shuttlecock speed (km.h-1) & & $121.0 \pm 4.2$ & $123.3 \pm 4.8$ & 0.81 & $2.1 \pm 0.6$ & 2.2 & & $122.8 \pm 3.8$ & $123.8 \pm 4.9$ & 0.33 & $2.8 \pm 0.8$ & 3.8 \\
\hline Precision (/60) & & $21.3 \pm 1.7$ & $21.7 \pm 2.8$ & 0.30 & $8.6 \pm 3.6$ & 2.0 & & $20.7 \pm 1.8$ & $23.7 \pm 1.0$ & 0.53 & $9.7 \pm 5.2$ & 1.1 \\
\hline UT & 10 & & & & & & 10 & & & & & \\
\hline Shuttlecock speed (km.h-1) & & $101.7 \pm 5.3$ & $102.1 \pm 6.2$ & 0.78 & $2.4 \pm 1.4$ & 3.0 & & $101.7 \pm 5.3$ & $102.8 \pm 9.2$ & 0.63 & $4.0 \pm 2.3$ & 4.9 \\
\hline Precision (/60) & & $18.4 \pm 3.0$ & $18.6 \pm 3.4$ & 0.42 & $12.2 \pm 7.3$ & 2.5 & & $18.4 \pm 3.0$ & $17.4 \pm 2.2$ & -0.14 & $14.1 \pm 4.6$ & 2.7 \\
\hline
\end{tabular}


643 Table 2. Intra- and inter-reliability for shuttlecock speed and precision for high trained (HT), moderate trained (MT), low trained (LT) and untrained 644 group (UT). T1: average of the first series of the first session. T2: average of the second series of the first session. T3: average of the first series of 645 the second session. ICC: intraclass correlation coefficient; CV: coefficient of variation; SEM: standard error of measurement. Values are mean \pm 646 SD. 


\section{$647 \quad$ Figure captions}

648

649 Figure 1. Top view of the device used during the specific test

650 (panel A). The black cross represents the position of the player

651 to hit the shuttlecock. Black areas represent "accuracy" (3 pts)

652 and grey areas represent "consistency" (2 Pts). Top view of the

653 device used to induce fatigue (panel B). The grey square

654 represents the replacement of the player after each lunge.

655

656 Figure 2. Height of the 100 countermovement jumps of the

657 fatiguing protocol (10 sets of 10 repetitions). $* * *$ significant

658 difference between set 1 and the other sets $(\mathrm{P}<0.001$, mean

659 height of the sets). Data are presented as means \pm SD.

660

661 Figure 3. Effects of muscle fatigue on smash stroke parameters

662 related to the badminton performance test: ball speed (panel A),

663 precision (panel B) and distribution (panel C). ** and $* * *$

664 significant differences between pre fatigue and post fatigue

$665(\mathrm{P}<0.01, \mathrm{P}<0.001$, respectively $) . \dagger$ trend difference between pre

666 and post $(0.05<\mathrm{P}<0.1)$. Data are presented as means $\pm \mathrm{SD}$. 